\title{
Ultra-Reliable and Low-Latency Vehicular Communication: An Active Learning Approach
}

\author{
Mohamed K. Abdel-Aziz, Student Member, IEEE, Sumudu Samarakoon, Member, IEEE, Mehdi Bennis, Senior \\ Member, IEEE, and Walid Saad, Fellow, IEEE
}

\begin{abstract}
In this letter, an age of information (AoI)-aware transmission power and resource block (RB) allocation technique for vehicular communication networks is proposed. Due to the highly dynamic nature of vehicular networks, gaining a prior knowledge about the network dynamics, i.e., wireless channels and interference, in order to allocate resources, is challenging. Therefore, to effectively allocate power and RBs, the proposed approach allows the network to actively learn its dynamics by balancing a tradeoff between minimizing the probability that the vehicles' AoI exceeds a predefined threshold and maximizing the knowledge about the network dynamics. In this regard, using a Gaussian process regression (GPR) approach, an online decentralized strategy is proposed to actively learn the network dynamics, estimate the vehicles' future AoI, and proactively allocate resources. Simulation results show a significant improvement in terms of AoI violation probability, compared to several baselines, with a reduction of at least $50 \%$.
\end{abstract}

Index Terms-Gaussian process regression (GPR), ultrareliable low-latency communication (URLLC), age of information (AoI), V2X.

\section{INTRODUCTION}

Vehicle-to-vehicle (V2V) communication is an important application in 5G and beyond networks. Time-critical V2V safety applications require ultra-reliable low-latency communication (URLLC), in which the freshness of vehicles' status update is crucial. A relevant metric to quantify information freshness is the age of information (AoI). AoI is defined as the time elapsed since the generation of the last received status update [1], [2]. Providing reliability guarantees in terms of AoI is essential for such applications. In fact, if future AoI can be reliably estimated, proactive transmission power and resource block (RB) allocation can be carried out to ensure that the reliability, defined as the probability of the future AoI exceeding a threshold, is minimized. However, for an accurate estimation of future AoI, knowledge of the network dynamics, i.e., wireless channels and interference, is required. Recently, several works have studied the optimization of AoI in vehicular networks [3]-[5]. In [3] and [4], the network dynamics are assumed to be known and in [5], they are estimated in a centralized manner without any consideration of future AoI. Yet, in a URLLC setting [6], [7], reliably

This work was supported in part by the Academy of Finland project CARMA, and 6Genesis Flagship (grant no. 318927), in part by the INFOTECH project NOOR, in part by the Office of Naval Research (ONR) under MURI Grant N00014-19-1-2621, and in part by the Kvantum Institute strategic project SAFARI.

M. K. Abdel-Aziz and S. Samarakoon are with the Centre for Wireless Communications, University of Oulu, 90014 Oulu, Finland (e-mails: mohamed.abdelaziz@oulu.fi; sumudu.samarakoon@oulu.fi).

M. Bennis is with the Centre for Wireless Communications, University of Oulu, 90014 Oulu, Finland, and also with the Department of Computer Engineering, Kyung Hee University, Yongin 446-701, South Korea (e-mail: mehdi.bennis@oulu.fi).

W. Saad is with the Department of Electrical and Computer Engineering, Virginia Tech, Blacksburg, VA 24061, USA (e-mail: walids@ vt.edu)

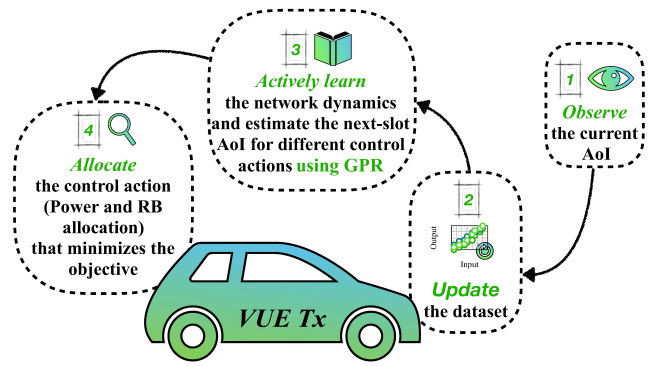

Figure 1. Illustration of the proposed solution.

learning and estimating the network dynamics with minimum communication overhead is desirable. Due to the highly dynamic nature of vehicular networks, gaining knowledge a priori about the network dynamics is challenging. In this regard, a viable solution is to develop an online decentralized strategy to actively learn ${ }^{1}$ the network dynamics and allocate resources accordingly to ensure reliability [8]. One possible approach is based on model predictive control (MPC) [9], which allows to control dynamic systems while ensuring the optimality of the system performance. However, MPC requires an accurate mathematical model of the dynamic system a priori, which is unrealistic in vehicular networks. One alternative is based on a Gaussian process regression (GPR) approach, leveraging its modeling flexibility and robustness to overfitting [8]. Unlike Gaussian mixture model (GMM), which is a parametric model and suffers from overfitting, GPR is a non-parametric Bayesian learning approach which means that it is not limited by a functional form [10].

The main contribution of this paper is to develop a novel approach, based on GPR, for enabling URLLC among vehicles by actively learning the system dynamics, estimating the vehicles' future AoI, and proactively allocating transmission power and RBs, in an online decentralized manner, as illustrated in Fig. 1. The main objective is to trade off between maximizing reliability by minimizing the probability that the AoI exceeds a predefined threshold and maximizing the knowledge gain about the local network dynamics. Simulation results show a significant gain in terms of maximizing reliability, compared to other baselines, with more than a 2fold improvement. Moreover, our numerical results show that accurately estimating the future AoI improves the overall performance in terms of minimizing the AoI violation probability.

\section{SYSTEM MODEL}

Consider a V2V communication network consisting of a set $\mathcal{K}$ of $K$ VUE transmitter-receiver pairs under the coverage of a single roadside unit (RSU), assuming a Manhattan mobility

\footnotetext{
${ }^{1}$ Active learning is a paradigm in which the learning process is directed to provide the next-best data input, e.g., resource allocation in our case, that optimizes an objective according to the historical data.
} 
model $^{2}$. The VUE pairs share a set $\mathcal{N}$ of $N$ orthogonal RBs with equal bandwidth $W$. The communication timeline is slotted with a slot duration of $\tau$, with $t$ being the slot index. For each VUE pair $k, \boldsymbol{P}_{k}(t)=\left[P_{k}^{n}(t)\right]_{n=1}^{N} \in \mathcal{P}$ is an $N$-dimensional power allocation vector over different RBs, with $\mathcal{P}$ being the set of all feasible power allocations and $P_{k}^{n}(t) \in\left\{0, \frac{p}{L}, \frac{2 p}{L}, \ldots, p\right\}$, where $p$ is the maximum allowed transmission power per RB and $L+1$ is the number of available power levels ${ }^{3}$. Moreover, the allocated transmission power is subject to $\sum_{n \in \mathcal{N}} P_{k}^{n}(t) \leq P_{\max }$, where $P_{\max }$ is the total power budget, which is assumed equal for all VUE pairs. Furthermore, each VUE transmitter $k$ has a queue buffer to store the data destined to its receiver whose queue dynamics are given by: $Q_{k}(t+1)=\max \left(Q_{k}(t)-R_{k}(t), 0\right)+A$, where $Q_{k}(t)$ is the queue length of VUE pair $k$ at time slot $t, A$ is the periodic packet arrival rate per slot, and $R_{k}(t)$ is the transmission rate of each VUE pair $k$ at time slot $t$ (in packets per slot), which is given by,

$$
R_{k}(t)=\frac{\tau}{Z} \sum_{n \in \mathcal{N}} W \log _{2}\left(1+\frac{P_{k}^{n}(t) h_{k k}^{n}(t)}{N_{0} W+I_{k}^{n}(t)}\right),
$$

where $Z$ is the packet length in bits, and $N_{0}$ is the power spectral density of the additive white Gaussian noise. Here, $I_{k}^{n}(t)=\sum_{k^{\prime} \in \mathcal{K} \backslash\{k\}} P_{k^{\prime}}^{n}(t) h_{k^{\prime} k}^{n}(t)$ is the aggregate interference from other VUEs at the receiver of VUE pair $k$ over RB $n$, and $h_{k k}^{n}(t)$ is the channel gain from the transmitter of VUE pair $k$ to its receiver over RB $n$. We use the realistic V2V channel model of [11] in which, depending on the location of the VUE transmitter and receiver, the channel model is categorized into three types: Line-of-sight, weak-line-of-sight, and non-line-of-sight.

Real-time status updates are critical for $\mathrm{V} 2 \mathrm{~V}$ safety applications, whose freshness can be characterized using the AoI metric [1], defined as: $\triangle_{k}(t) \triangleq \tau t-\gamma_{k}(t)$, where $\triangle_{k}(t)$ denotes the AoI of VUE pair $k$ at the beginning of time slot $t$ and $\gamma_{k}(t)$ is the generation instant of the last status update received by VUE receiver $k$ at/or just before the beginning of time slot $t$. Note that, the index of the last received status update depends on $R_{k}(t-1)$, which, according to (1), depends on the channel gain, interference, and allocated power. Therefore, $\triangle_{k}(t+1)$ can be expressed as:

$$
\triangle_{k}(t+1)=f_{k}\left(\triangle_{k}(t), \boldsymbol{P}_{k}(t)\right), \forall k \in \mathcal{K},
$$

where $f_{k}(\cdot)$ is a nonlinear dynamic system for VUE pair $k$ which represents the local network dynamics, i.e. wireless channels and interference. In this dynamic system, $\triangle_{k}(t) \in$ $\mathbb{R}^{+}$and $\boldsymbol{P}_{k}(t) \in \mathcal{P}$ represent the state of the system and the control action at time slot $t$, respectively. $f_{k}: \mathbb{R}^{+} \times \mathcal{P} \rightarrow$ $\mathbb{R}^{+}$maps the control actions to the state of the system [8]. Without any prior knowledge about the channel $h_{k k}^{n}(t)$ and interference $I_{k}^{n}(t)$, and due to the highly non-stationary nature of a vehicular network, the relationship $f_{k}(\cdot)$ is unknown a priori for every VUE pair $k$. Thus, $f_{k}(\cdot)$ needs to be reliably learned online from historical data. Jointly controlling and actively learning $f_{k}(\cdot)$ is crucial to reliably estimate the future AoI and allocate resources.

\footnotetext{
${ }^{2} \mathrm{~A}$ transmitter periodically shares its status with a receiver in a vehicular rear-end collision aviodance scenario.

${ }^{3}$ Note that $P_{k}^{n}(t)=0$ implies that RB $n$ is not allocated to VUE pair $k$.
}

Estimating $f_{k}(\cdot)$ using a set of historical data points is a regression problem. For our model, GPR is used as the regression method, which is a class of Bayesian nonparametric machine learning models. GPR does not involve any black-box operations, and has a promising potential in improving prediction accuracy. Moreover, Gaussian processes (GPs) provide an elegant mathematical method for regression accompanied with the full predictive distribution, which is important for establishing confidence intervals [8], [12]. In this view, we can rewrite (2), for notational simplicity, as $y_{i}=f_{k}\left(\boldsymbol{x}_{i}\right)$, where $\boldsymbol{x}_{i}=\left[\triangle_{k}(i), \boldsymbol{P}_{k}(i)\right]$ represents the $i^{\text {th }}$ input and $y_{i}=\triangle_{k}(i+1)$ represents the corresponding scalar output. GPR is a nonlinear regressor that expresses the inputoutput relation by assuming that $f_{k}(\cdot)$ a priori follows a $\mathrm{GP}^{4}$ [12]. For any finite dataset $\mathcal{D}_{k}=\left\{\boldsymbol{x}_{i}, y_{i}\right\}_{i=1}^{M}$, where $M$ is the dataset size, a GP becomes a multidimensional Gaussian defined by its mean (zero in our case, for simplicity) and covariance matrix, $\boldsymbol{C} \triangleq\left[c\left(\boldsymbol{x}_{i}, \boldsymbol{x}_{j}\right)\right]_{i j}, \forall \boldsymbol{x}_{i}, \boldsymbol{x}_{j} \in \mathcal{D}_{k}$. For a general input $\boldsymbol{x}_{*}$ and for a given $\mathcal{D}_{k}$, GPR provides a full statistical description of $y_{*}$, namely $\operatorname{Pr}\left\{y_{*} \mid \boldsymbol{x}_{*}, \mathcal{D}_{k}\right\}$, which can be computed using the standard tools of Bayesian statistics leading to $\left.y_{*}\right|_{\boldsymbol{x}_{*}, \mathcal{D}_{k}} \sim \mathscr{N}\left(\mu_{y_{*}}, \sigma_{y_{*}}^{2}\right)$, where $\mathscr{N}\left(\mu_{y_{*}}, \sigma_{y_{*}}^{2}\right)$ is the normal distribution with mean $\mu_{y_{*}}$ and variance $\sigma_{y_{*}}^{2}$. Moreover,

$$
\begin{aligned}
\mu_{y_{*}} & =\boldsymbol{c}_{*}^{\mathrm{T}} \boldsymbol{C}^{-1} \boldsymbol{y}, \\
\sigma_{y_{*}}^{2} & =c\left(\boldsymbol{x}_{*}, \boldsymbol{x}_{*}\right)-\boldsymbol{c}_{*}^{\mathrm{T}} \boldsymbol{C}^{-1} \boldsymbol{c}_{*},
\end{aligned}
$$

with $\boldsymbol{c}_{*} \triangleq\left[c\left(\boldsymbol{x}_{*}, \boldsymbol{x}_{1}\right) \cdots c\left(\boldsymbol{x}_{*}, \boldsymbol{x}_{M}\right)\right]^{\mathrm{T}}$ and $\boldsymbol{y}=\left[y_{1} \cdots y_{M}\right]^{\mathrm{T}}$ [12]. Here, the mean given in (3) is the estimate of $y_{*}$. Note that the GPR computation complexity grows cubically with the dataset size $M$ [10]. However, due to the dynamic nature of vehicular networks, fixing the maximum size of the dataset by discarding old samples (i.e. history), can help tackle the computational complexity without affecting the GPR estimation performance.

A GP is completely defined by its covariance function $c\left(\boldsymbol{x}_{i}, \boldsymbol{x}_{j}\right)$. To accurately estimate future measures and their distributions, a covariance function that fits with the nature of the system has to be selected. In this regard, a Matérn class covariance function [13] has been selected for learning $f_{k}(\cdot)$ :

$c\left(\boldsymbol{x}_{i}, \boldsymbol{x}_{j}\right)=h^{2} \frac{2^{1-\nu}}{\Gamma(\nu)}\left(2 \sqrt{\nu} \frac{\left|\boldsymbol{x}_{i}-\boldsymbol{x}_{j}\right|}{\lambda}\right)^{\nu} \mathbb{B}\left(2 \sqrt{\nu} \frac{\left|\boldsymbol{x}_{i}-\boldsymbol{x}_{j}\right|}{\lambda}\right)$,

where $\Gamma(\cdot)$ is the standard Gamma function and $\mathbb{B}(\cdot)$ is the modified Bessel function of second order. The Matérn class covariance function includes both the exponential autocorrelation function when $\nu=0.5$ and the Gaussian autocorrelation function as a limiting case when $\nu \rightarrow \infty$. Therefore, the Matérn class offers us a flexibility to strike a balance between these two extremes, thus it is well suited for our application. The variables $h, \lambda$ and $\nu$ are the hyperparameters of the covariance function. The hyperparameters determine the shape of the covariance function which need to be tuned to fit the observed dataset $\mathcal{D}_{k}$. This is done by maximizing the marginal likelihood of the GP [10].

\footnotetext{
${ }^{4}$ Note that this assumption does not mean that the underlying process is precisely Gaussian, but GP can still be used as a maximum entropy process, for a given covariance function.
} 
Table I

SimUlation PARAMETERS

\begin{tabular}{|c|c|c|c|c|c|}
\hline Para. & Value & Para. & Value & Para. & Value \\
\hline$K$ & 20 & $N$ & 20 & $\tau$ & $3 \mathrm{~ms}$ \\
$W$ & $180 \mathrm{kHz}$ & $N_{0}$ & $-174 \mathrm{dBm} / \mathrm{Hz}$ & $p$ & $10 \mathrm{dBm}$ \\
$P_{\max }$ & $17 \mathrm{dBm}$ & $L$ & 1 & arrival rate & $2.5 \mathrm{Mbps}$ \\
$Z$ & $500 \mathrm{Byte}$ & $\alpha_{c}$ & 1 & $\alpha_{i}$ & 100 \\
$d$ & $10 \mathrm{~ms}$ & $M$ & 200 & & \\
\hline
\end{tabular}

Actively learning $f_{k}(\cdot)$, by choosing the next-best input $\boldsymbol{x}_{*}$ that optimizes an objective, would be beneficial in vehicular networks due to its high non-stationary nature. The choice of this objective and the problem formulation is discussed next.

\section{AOI-AwARe Power AND RB Allocation}

Each VUE pair has two main objectives. First, it seeks to improve the reliability of the status updates and second, it needs to enhance its knowledge about the system dynamics. Improving the status updates reliability is captured by choosing the resources allocation that minimizes $\operatorname{Pr}\left\{\hat{\triangle}_{k}(t+1)>d\right\}$. Here, the next-slot AoI is estimated using GPR and thus, $\hat{\triangle}_{k}(t+1)$ is a Gaussian distributed random variable with mean and variance given by (3) and (4), respectively. Moreover, enhancing the knowledge about $f_{k}(\cdot)$ is tantamount to choosing the allocation that maximizes the estimated AoI variance, $\sigma_{\hat{\Delta}_{k}(t+1)}^{2}[8$, Corollary 5.4]. Formally, actively learning $f_{k}(\cdot)$ by optimizing the tradeoff between improving status update reliability (exploitation) and enhancing the system dynamics knowledge (exploration) of VUE pair $k$ at time $t$ can be posed as follows:

$$
\min _{\boldsymbol{P}_{k}(t) \in \mathcal{P}} \alpha_{c} \operatorname{Pr}\left\{\hat{\triangle}_{k}(t+1)>d\right\}-\alpha_{i} \sigma_{\hat{\triangle}_{k}(t+1)}^{2}
$$

subject to (2),

where $\alpha_{c}$ and $\alpha_{i}$ are non-negative weighting factors that capture the exploitation-exploration tradeoff. A control action $\boldsymbol{P}_{k}(t) \in \mathcal{P}$ that enhances the knowledge about the unknown system $f_{k}($.$) may not necessarily be the one that improves the$ reliability $\operatorname{Pr}\left\{\hat{\triangle}_{k}(t+1)>d\right\}$. Thus, the choice of $\alpha_{c}$ and $\alpha_{i}$ affect the system performance, as will be shown in Section IV. Note that, since $\hat{\triangle}_{k}(t+1)$ follows a Gaussian distribution, then $\operatorname{Pr}\left\{\hat{\triangle}_{k}(t+1)>d\right\}=\frac{1}{2} \operatorname{erfc}\left(\frac{d-\mu_{\hat{\Delta}_{k}(t+1)}}{\sqrt{2 \sigma_{\widehat{\triangle}_{k}(t+1)}^{2}}}\right)$.

Actively learning $f_{k}(\cdot), \forall k \in \mathcal{K}$ at a central entity, i.e., RSU, incurs huge communication overhead due to exchanging the AoI of each VUE pair and the resources allocation decisions between the RSU and all VUE pairs at every time slot $t$. Thus, a decentralized AoI estimation and resources allocation approach is proposed ${ }^{5}$. For a given VUE pair $k$, Algorithm 1, which is given from [8], provides the steps to obtain the solution of (5).

\section{Simulation Results}

We assume a $250 \times 250 \mathrm{~m}^{2}$ area of a Manhattan mobility model as in [14]. We set the average vehicle speed to $60 \mathrm{~km} / \mathrm{h}$, and we choose a time-varying distance between each transmitter-receiver pair while maintaining an average distance of $15 \mathrm{~m}$ [15], with a simulation duration of $T=$

\footnotetext{
${ }^{5} \mathrm{~A}$ VUE transmitter only requires the state (AoI) of its receiver.
}
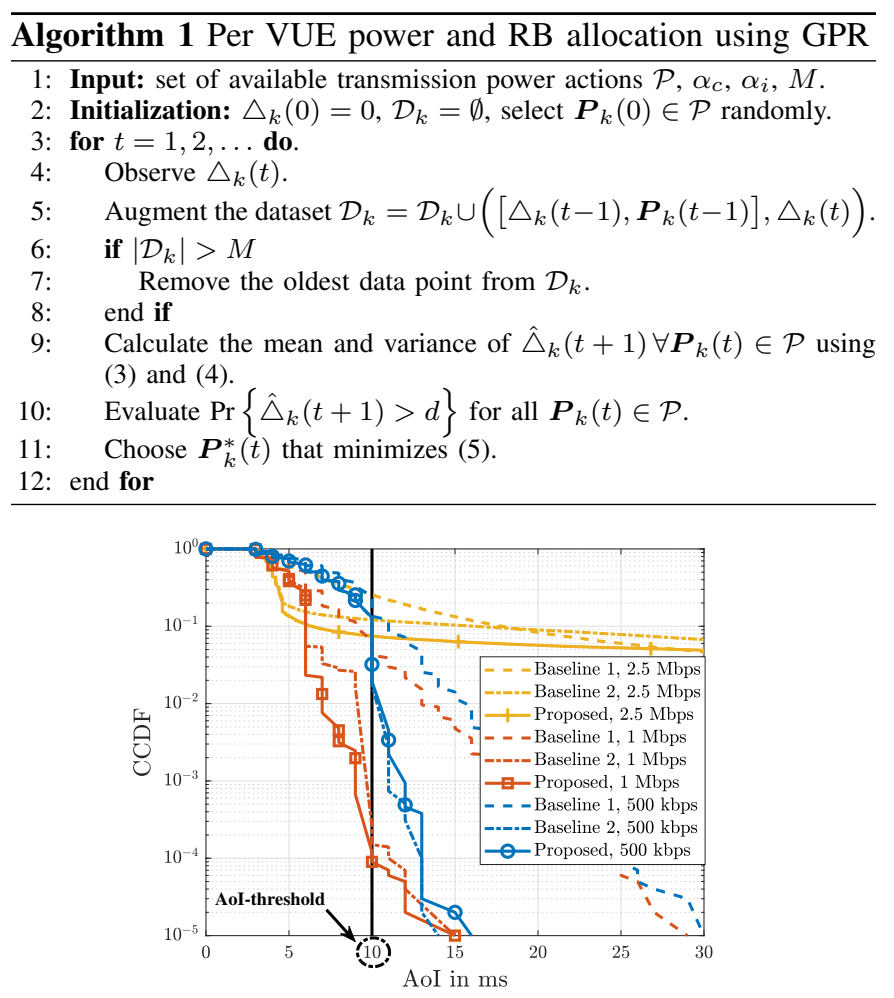

Figure 2. CCDF of the AoI for various RB allocation schemes and different arrival rates with $K=20$ and $M=200$.

5000 time slots. Table I lists all main simulation parameters. The GPML toolbox has been used to implement GPR [16]. A performance comparison is carried out between different schemes: i) Baseline 1: each VUE pair allocates its transmission power randomly without learning the system dynamics, i.e. wireless channel and interference, ii) Baseline 2 (GPRbased Allocation with no exploration): a greedy approach of our proposed scheme where each VUE pair actively learns its system dynamics $f_{k}$ using GPR and subsequently solves (5) with $\alpha_{i}=0$, and iii) Proposed: GPR-based power and RB allocation with $\alpha_{i}=100$.

Fig. 2 compares the AoI of the different schemes in terms of complementary cumulative distribution function (CCDF) for different status updates' arrival rates. Note that, due to the learning of system dynamics $f_{k}$, the GPR-based allocation schemes (Baseline 2 and Proposed) outperform Baseline 1 for all of the considered arrival rates, in terms of the AoI violation probability, i.e. $\operatorname{Pr}\{\triangle(t)>d\}$. In this regard, the AoI violation probability is reduced by at least $52 \%, 85 \%$, and $99.6 \%$, when the arrival rate is $2.5 \mathrm{Mbps}, 500 \mathrm{kbps}$, and $1 \mathrm{Mbps}$, respectively. Moreover, Fig. 2 shows that the AoI violation probability when the arrival rate is $1 \mathrm{Mbps}$ is significantly lower than the AoI violation probability when the arrival rate is $500 \mathrm{kbps}$ or $2.5 \mathrm{Mbps}$. This means that the arrival rate of status updates plays a significant role in determining the performance of the system. Furthermore, it should be noted that exploration can further reduce the AoI violation probability. This reduction is more pronounced when the arrival rates are $1 \mathrm{Mbps}$ and $2.5 \mathrm{Mbps}$.

In Fig. 3, the next-slot AoI estimation performance and the AoI violation probability are shown for the different dataset sizes $M$ used for active learning. Here, the shaded area 


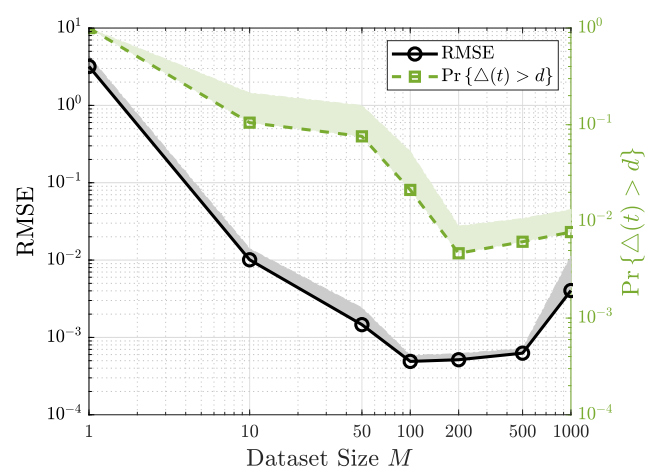

Figure 3. RMSE and AoI violation probability for different dataset sizes $M$ with $K=20, \alpha_{\mathrm{c}}=1$ and $\alpha_{\mathrm{i}}=0$

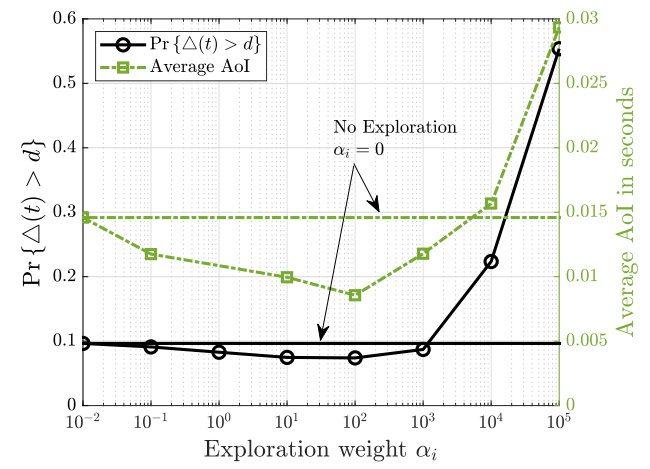

Figure 4. Exploration vs AoI violation probability and average AoI.

highlights the upper $95 \%$ confidence interval. The estimation performance is measured using the root mean square error (RMSE) metric, which is calculated per VUE pair $k$ as $\sqrt{\sum_{t}\left(\mu_{\widehat{\Delta}_{k}(t+1)}-\triangle_{k}(t+1)\right)^{2} / T}$. Note that as $M$ increases, the next-slot AoI estimation becomes more accurate, reducing the RMSE, up until $M \approx 100$. However, beyond $M=100$, RMSE increases and becomes worse at $M=1000$. This corroborates the fact that leveraging more history about the system is not always beneficial and may hurt the overall performance. This is due to the fast varying vehicular environment, outdated observations become uncorrelated, yielding poor prediction accuracy. Therefore, the main drawback of GPR, being computationally heavy, diminishes because accurately estimating the next-slot AoI would not require largesized datasets. Furthermore, Fig. 3 shows that accurate AoI estimation improves decision making in terms of minimizing the AoI violation probabilities. Following the RMSE trend, the probability of AoI violation decreases when using up to $M=200$ data samples. The uncorrelated and outdated data samples in scenarios with $M>200$ lead to poor prediction accuracy thereby increasing the AoI violations probability.

In Fig. 4, we study the impact of the exploration parameter $\alpha_{i}>0$ on the average AoI and AoI violation probabilities. Fig. 4 shows that, as the exploration weight $\alpha_{i}$ increases up to $10^{2}$, the AoI violation probability and the average AoI decrease. This means that exploration allows each VUE pair to sample better control decisions thus enhancing the performance. However, further increasing $\alpha_{i}>10^{2}$ results in a performance degradation, in terms of an increased AoI violation probability and increased average AoI. The reason is that when the exploration weight is high, each VUE pair will be biased towards choosing the control action that provides more knowledge about the system instead of minimizing the AoI violation probability. Henceforth, a balance between exploration and exploitation needs to be taken into account.

\section{CONCLUSION}

In this paper, we have studied the problem of allocating transmission power and RBs in vehicular networks under uncertainty. The main objective is to balance a tradeoff between minimizing the probability of the predicted AoI exceeding a certain threshold and maximizing the amount of knowledge learned about the system dynamics in an online manner. In this regard, GPR is used to actively learn the network dynamics and estimate the future AoI, and a decentralized allocation approach is proposed. Simulation results have shown a significant improvement in terms of AoI violation probability, compared to other baselines. Finally, our results have also shown that a balance between exploration and exploitation is important to yield the best performance in terms of AoI violation probability. Thus, finding the optimal dataset size and exploration-exploitation weights to ensure a reliability target could be an interesting topic for future work.

\section{REFERENCES}

[1] S. Kaul, M. Gruteser, V. Rai, and J. Kenney, "Minimizing Age of Information in Vehicular Networks," in Proc. IEEE Commun. Soc. Conf. on Sensor, Mesh and Ad Hoc Commun. and Netw., Salt Lake City, UT, USA, Jun. 2011, pp. 350-358.

[2] M. K. Abdel-Aziz, C. Liu, S. Samarakoon, M. Bennis, and W. Saad, "Ultra-Reliable Low-Latency Vehicular Networks: Taming the Age of Information Tail," in Proc. IEEE Global Commun. Conf., Abu Dhabi, UAE, Dec 2018, pp. 1-7.

[3] A. O. Al-Abbasi and V. Aggarwal, "Joint Information Freshness and Completion Time Optimization for Vehicular Networks," CoRR, vol. abs/1811.12924, 2018. [Online]. Available: http://arxiv.org/abs/1811.12924

[4] S. Zhang, J. Li, H. Luo, J. Gao, L. Zhao, and X. S. Shen, "Towards Fresh and Low-Latency Content Delivery in Vehicular Networks: An Edge Caching Aspect," in 2018 10th International Conference on Wireless Communications and Signal Processing (WCSP), Hangzhou, China, Oct 2018, pp. 1-6.

[5] Y. Ni, L. Cai, and Y. Bo, "Vehicular Beacon Broadcast Scheduling Based on Age of Information (AoI)," China Communications, vol. 15, no. 7, pp. 67-76, July 2018.

[6] M. Angjelichinoski, K. F. Trillingsgaard, and P. Popovski, "A Statistical Learning Approach to Ultra-Reliable Low Latency Communication," IEEE Trans. on Commun., pp. 1-1, 2019.

[7] W. Saad, M. Bennis, and M. Chen, "A Vision of 6G Wireless Systems: Applications, Trends, Technologies, and Open Research Problems," IEEE Network, to appear, 2019.

[8] T. Alpcan and I. Shames, "An Information-Based Learning Approach to Dual Control," IEEE Trans. Neural Netw. Learn. Syst., vol. 26, no. 11, pp. 2736-2748, Nov. 2015.

[9] J. B. Rawlings, "Tutorial Overview of Model Predictive Control," IEEE Control Systems Magazine, vol. 20, no. 3, pp. 38-52, June 2000.

[10] C. E. Rasmussen and C. K. Williams, Gaussian Processes for Machine Learning. The MIT Press, 2005.

[11] M. Abdulla and H. Wymeersch, "Fine-Grained vs. Average Reliability for V2V Communications around Intersections," in Proc. IEEE Global Commun. Conf. Workshops, Singapore, Dec. 2017, pp. 1-5.

[12] F. Perez-Cruz, S. V. Vaerenbergh, J. J. Murillo-Fuentes, M. LazaroGredilla, and I. Santamaria, "Gaussian Processes for Nonlinear Signal Processing: An Overview of Recent Advances," IEEE Signal Process. Mag., vol. 30, no. 4, pp. 40-50, Jul. 2013.

[13] M. L. Stein, Interpolation of Spatial Data. Springer New York, 1999.

[14] C.-F. Liu and M. Bennis, "Ultra-Reliable and Low-Latency Vehicular Transmission: An Extreme Value Theory Approach," IEEE Commun. Lett., vol. 22, no. 6, pp. 1292-1295, Jun. 2018.

[15] ETSI TR 138 913, "5G; Study on Scenarios and Requirements for Next Generation Access Technologies," V14.2.0, May 2017.

[16] C. E. Rasmussen and H. Nickisch, "Gaussian processes for machine learning (GPML) toolbox," J. Mach. Learn. Res., Nov. 2010. 